\title{
Inertial microfluidic purification of floating cancer cells for drug screening and three-dimensional tumour models
}

Jun Zhang ${ }^{a *^{+}}$, Naveen Chintalaramulu ${ }^{\mathrm{b} *^{+}}$, Raja Vadivelu ${ }^{\mathrm{a}, \mathrm{c}+}$, Hongjie An ${ }^{\mathrm{a}}$, Dan Yuan ${ }^{\mathrm{d}}$, Jing Jin $^{\text {a }}$, Chin Hong Ooi ${ }^{\text {a }}$, Ian Edwin Cock ${ }^{\text {b,e }}$, Weihua Li $^{\text {f }}$, Nam-Trung Nguyen ${ }^{\text {a* }}$

${ }^{a}$ Queensland Micro and Nanotechnology Centre, Griffith University, Brisbane QLD 4111, Australia, Fax: +61 07373 58021; Tel: +61 (07) 373 53921; E-mail: jun.zhang@griffith.edu.au; nam-trung.nguyen@griffith.edu.au; b School of Environment and Science, Nathan Campus, Griffith University, 170 Kessels Road, Brisbane, QLD, 4111, Australia; E-mail: naveen.chintalaramulu@griffithuni.edu.au;

${ }^{c}$ Department of Chemical System Engineering, The University of Tokyo, Tokyo 113-8656, Japan

${ }^{\mathrm{d}}$ Department of Chemistry, University of Tokyo, Tokyo, 113-0033, Japan

e Environmental Futures Research Institute, Nathan Campus, Griffith University, 170 Kessels Road, Brisbane, QLD, 4111, Australia

${ }^{\mathrm{f}}$ School of Mechanical, Materials and Mechatronic Engineering, University of Wollongong, Wollongong, NSW 2522, Australia

+ These authors contributed equally to this work.

\section{ABSTRACT}

Floating cancer cells can survive the programmed death anoikis process after detaching from the extracellular matrix for the anchorage-dependent cells. Purification of viable floating cancer cells is essential for many biomedical studies, such as drug screening and cancer model development. However, the floating cancer cells are mixed with dead cells and debris in the medium supernatant. In this paper, we developed an inertial microfluidic device with sinusoidal microchannels to continuously remove dead cells and debris from viable cells. First, we characterized the differential inertial focusing properties of polystyrene beads in the devices. Then, we investigated the effects of flow rate on inertial focusing of floating MDAMB-231 cells. At an optimal flow condition, purification of viable cells was performed and the purity of live cells was increased significantly from $19.9 \%$ to $76.6 \%$, with a recovery rate of $69.7 \%$. After separation, we studied and compared the floating and adherent MDA-MB231 cells in terms of cell proliferation, protrusive cellular structure, and the expression of cyclooxygenase (Cox-2) which is related to epithelial-mesenchymal transition (EMT) changes. Meanwhile, drug screening of both floating and adherent cancer cells was conducted using a chemotherapeutic drug - doxorubicin (Dox). The results revealed that the floating cancer cells possess 30 -fold acquired chemoresistance as compared to the adherent cancer 
cells. Furthermore, a three-dimensional (3D) double-cellular co-culture model of human mammary fibroblasts (HMF) spheroid and cancer cells using the floating liquid marble technique was developed.

\section{INTRODUCTION}

Cancer metastasis is one of the hallmarks of cancer ${ }^{1}$ and the cause of approximately $90 \%$ of cancer-related deaths ${ }^{2,3}$. Metastasis requires the dissociation of cancer cells from the primary tumour site to enable distant colonization ${ }^{4,5}$. Generally, the interaction of normal epithelial cells with extracellular matrix proteins is mediated by integrins, a class of transmembrane receptors. Through their interaction with the extracellular matrix (ECM) proteins, integrins relay antiapoptotic signals to the cells thereby enhancing survival, proliferation and migration 6-9. Upon detachment from the ECM, these cells undergo a distinct form of apoptosis termed 'anoikis' ${ }^{10}$. In contrast, cancer cells demonstrate anoikis resistance as a prerequisite for metastasis ${ }^{11}$. Paoli et al. ${ }^{12}$ speculated that anoikis resistance may be caused by the hyperactivation of intracellular signalling cascades that induce changes in the integrin expression and enable the activation of phosphoinositide 3-kinase (PI3k)/Akt pathway. Furthermore, several studies have revealed a correlation between anchorage-independent growth and the metastatic potential of cancer cells ${ }^{13-15}$.

Breast cancer cell lines including non-metastatic MCF7 and highly metastatic MDAMB-231 cells are widely used as breast cancer models to study the metastatic potential of the disease ${ }^{16-18}$. Notably, MDA-MB-231 cells are characterized by the absence of estrogen, progesterone and human epidermal growth factor receptors $\mathrm{ER}^{-} / \mathrm{PR}^{-} / \mathrm{HER}^{2}$, termed triplenegative (TN). Interestingly, TN breast cancer is a highly metastatic form of the disease with poor prognosis ${ }^{19}$. MDA-MB-231 cells were reported to detach from the ECM and form suspension colonies in an anchorage-independent manner ${ }^{20}$. Although the detached cells were originally thought to be dead, the viability of the floating MDA-MB-231 cells was detected using in-vitro assays ${ }^{21-23}$. Further investigation to characterize the floating cells may provide options for novel therapeutic strategies to induce anoikis sensitivity in these cells. Since the floating cells are present along with the massive number of dead cells and debris in the cell culture supernatant, purification of the viable floating cells is a prerequisite for all downstream studies. 
In an adherent cell culture, nonviable cells can be eliminated by their lack of attachment capability to the culture flask as compared to the viable cells. However, this feature is not available for cell cultures and production processes of floating cells ${ }^{24}$. Conventional labelbased cell sorting approaches such as fluorescence-activated cell sorting (FACS) and magnetic-activated cell sorting (MACS) requires modification of cells with expensive reagents and time-consuming processing procedures. Moreover, the use of fluorophores and antibodies may alter the viability and functionality of the cells ${ }^{25,26}$. Therefore, the ideal purification method should be label-free, accurate, sufficiently gentle to preserve cellular health and function, as well as capable of high-throughput operation. Several microfluidic technologies have been successfully developed to separate cells 24,27-34. However, these approaches are limited by factors such as sensitivity to $\mathrm{pH}$ and salt concentration, low throughput, severe channel clogging, encapsulation errors, sensitivity of surfactant, poor biocompatibility, or complicated nonlinear structure.

In this work, we proposed an efficient inertial microfluidic device with linear sinusoidal microchannels to continuously remove dead cells and debris from floating cancer cells (MDA-MB-231) in a label-free way. We first characterized the inertial focusing properties of microparticles in the device using polystyrene beads with the diameter ranging from $3 \mu \mathrm{m}$ to $20 \mu \mathrm{m}$. We then investigate the inertial separation performance of floating cancer cells using the developed device. After separation, the floating and adherent MDA-MB-231 cells were studied and compared in many aspects such as cell proliferation and cellular structures. Furthermore, both the floating and adherent MDA-MB-231 cells were tested with front-line chemotherapeutic drug doxorubicin (Dox) to explore the anchorage-independent survival of the floating cells. Finally, we proposed a three dimensional (3D) double-cellular co-culture model using the floating liquid marble technique.

\section{EXPERIMENTAL SECTION}

Microfluidic device design and fabrication. The inertial microfluidic device consists of curvilinear microchannels with 16 sinusoidal periods. The width of the microchannel is $200 \mu \mathrm{m}$, and the height is $50 \mu \mathrm{m}$. The radius of curvature is $250 \mu \mathrm{m}$. The device consists of one inlet and two outlets. For the inlet, an array of circular pillars with a gap of $50 \mu \mathrm{m}$ is implemented to remove large debris from the cell culture and release the cells before they enter the functioning microchannel. Two outlets collect large viable cells from the channel central area and smaller dead cells/debris from the sidewall region, respectively. All 
microchannels were fabricated by the standard photolithography and polydimethylsiloxane (PDMS) soft lithography techniques ${ }^{35,36}$.

Polystyrene particle preparation. To characterize particle inertial focusing and separation in the sinusoidal channel, seven sets of spherical polystyrene microbeads with diameters of $3 \mu \mathrm{m}$ (Phosphorex, Product No. 2105B, CV8\%), $5 \mu \mathrm{m}$ (Thermo Fisher Scientific, Product No. G0500, CV5\%), $8 \mu \mathrm{m}$ (Phosphorex, Product No. 129, CV10\%), $10 \mu \mathrm{m}$ (Thermo Fisher Scientific, Product No. G1000, CV5\%), $13 \mu \mathrm{m}$ (Thermo Fisher Scientific, Product No. 36-4, CV14\%), $15 \mu \mathrm{m}$ (Phosphorex, product No. 2228, CV8.31\%) and $20 \mu \mathrm{m}$ (Phosphorex, product No. 122, CV8.28\%) were respectively dispersed in deionized (DI) water and tested using the sinusoidal channel. The particle concentration in the suspensions is ranging from $\sim 10^{5}$ to $\sim 10^{6}$ counts $/ \mathrm{mL}$. Besides, Tween 20 (Sigma-Aldrich, Product No. P9416) was added to DI water as a surfactant at a weight ratio of $0.1 \%$ to avoid particle aggregation.

Experimental setup. The microfluidic device was placed on the stage of an inverted microscope (Nikon, Eclipse TS100) where a high-speed CCD camera (Photron, FASTCAM SA3) was mounted. Particle and cell suspensions contained in the disposable plastic syringes were infused into the microfluidic device by a syringe pump (Cetoni GmbH, neMESYS $290 \mathrm{~N})$. The high-speed camera captured the movement videos of single particles using an ultra-short exposure time. The open-source software ImageJ (National Institutes of Health USA) was used to post-process and analyse the captured videos. A colour-coded map was illustrated to show the distribution of particles at different flow conditions, and the colour in the map represents the normalized frequency of particles at the specific lateral position ${ }^{37}$.

Cell culture and staining. The human breast cancer (MDA-MB-231) and mammary fibroblasts cells used in the study were obtained from American Type Tissue Culture (ATCC, Manassas, VA, USA). Dulbecco's Modified Eagle Medium/Nutrient Mixture F-12 (DEME/F12), heat-inactivated fetal bovine serum (FBS), penicillin/streptomycin, and $0.25 \%$ trypsin-EDTA were purchased from the Gibco-Thermo Fisher Scientific (Waltham, MA, USA). Celltiter 96 Aqueous One Solution Reagent (MTS) was obtained from Promega Co. (Madison, WI, USA). Doxorubicin (Dox) was a generous gift from Assoc. Prof. Kathryn Tonissen, School of Environmental and Sciences, Griffith University, Australia. Dox was stored at a stock concentration of $10 \mathrm{mM}$ in dimethyl sulfoxide (DMSO) at $-20{ }^{\circ} \mathrm{C}$. Mitotracker Green FM was obtained from Thermo Fisher Scientific (Eugene, OR, USA). MDA-MB-231 and HMF cells were cultured in DEME/F-12, 10\% FBS and 1\% penicillin/ 
streptomycin in a humidified atmosphere at $37{ }^{\circ} \mathrm{C}, 5 \% \mathrm{CO}_{2}$. The supernatant medium in which the floating MDA-MB-231 cells were suspended was taken from the culture flask and processed by the microfluidic device. The number of live and dead cells were determined by a hemocytometry by the trypan blue as well as the acridine orange (AO) and propidium iodide (PI) cell viability kits (St. Louis, MO, USA). AO will stain all nucleated cells to generate green fluorescence, whereas PI can only enter dead cells with poor membrane integrity and stain all dead nucleated cells to generate red fluorescence.

Drug screening. Doxorubicin (Dox), also known as adriamycin, is a member of the anthracycline family of antibiotics. It is used as a front-line chemotherapeutic agent in the treatment of a wide range of malignancies, including breast cancer, osteosarcoma, Hodgkin's lymphoma, and leukaemia ${ }^{38-40}$. Dox acts by DNA intercalation ${ }^{41,42}$, inhibition of topoisomerase II ${ }^{43,44}$, and by the generation of free radicals ${ }^{45,46}$. It thereby elicits a range of cytotoxic effects in conjunction with antiproliferation resulting in DNA damage. The floating and adherent MDA-MB-231 cells were plated at a seeding density of $1 \times 10^{4}$ cells per well in $100 \mu \mathrm{L}$ of complete medium and incubated at $37^{\circ} \mathrm{C}$ for $24 \mathrm{~h}$. Post-seeding, the medium was removed and $100 \mu \mathrm{L}$ of fresh medium containing a varying concentration of Dox (0.01$100 \mu \mathrm{M}$ ) was added and incubated for $24 \mathrm{~h}$. Following the drug treatment, $20 \mu \mathrm{L}$ of MTS was added and further incubated for $1 \mathrm{~h}$ at $37^{\circ} \mathrm{C}$. The optical density (OD) was read at $490 \mathrm{~nm}$ with SpectraMax M3 (Molecular Devices). The percentage of DMSO in the medium was $0.1 \%(\mathrm{v} / \mathrm{v})$.

Generation of fibroblasts spheroid in liquid marble. To produce the liquid marbles, $10 \mu \mathrm{L}$ of the medium containing 10,000 human mammary fibroblasts (HMF) cells were used to dispense on a polytetrafluoroethylene (PTFE) (Sigma-Aldrich, product number 430935, the particle diameter of $1 \mu \mathrm{m}$ ) powder bed in a 6-well plate. The plate was gently rotated in a circular motion so that the PTFE particles could coat the surface of the liquid droplet and form the liquid marble. The liquid marbles were picked up using a customized pipette and placed in a 96-well-plate. In the wells, the marbles were floated by a double-layer configuration which is formed by a $100 \mu \mathrm{L}$ medium and a thin PTFE powder layer to increase the humidity and minimises the marble evaporation. Subsequently, the well-plate was placed in the incubator for 24 hours, and the HMF cells could freely associate and self-assemble into multicellular spheroids. 
Co-culture of cancer cells with fibroblast spheroids in liquid marble. Both floating and the adherent MDA-MB-231 cells were harvested from the culture flask, and the cell concentration was adjusted to $4 \times 10^{5}$ cells $/ \mathrm{mL}$. Both cells were stained by MitoTracker Green dye (Thermo Fisher Scientific, product number M7514) at a concentration of $15 \mathrm{ng} / \mathrm{mL}$, and incubated at $37{ }^{\circ} \mathrm{C}, 5 \% \mathrm{CO}_{2}$ for 30 mins. The cells were washed twice using culture medium and adjusted to a concentration of $4 \times 10^{5}$ cells $/ \mathrm{mL}$. Then, a cell medium of $5 \mu \mathrm{L}$ (containing 2,000 cells) was aspirated by a pipette and transplanted into the liquid marbles containing the HMF spheroids. The liquid marbles were picked up and placed in a 96-well-plate, where marbles were floated by the double-layer configuration. The cancer cells and HMF spheroids were co-cultured in the marbles for 24 hours. Subsequently, the marbles were broken by a needle, so that the HMF spheroids sedimented to the bottom of walls and the PTFE particles were removed from the supernatant for better observation of co-culture model of cancer cells and HMF spheroids.

\section{RESULTS AND DISCUSSION}

Characterization of Sinusoidal Microchannels. The separation principle of cells in this work is based on the differential focusing along the channel width in the sinusoidal microchannel, Figure 1(a). As the cells undergo the programmed cell death called apoptosis, they become smaller than the normal viable cells due to shrinkage and fragmentation ${ }^{32}$. The average size of the nonviable cells become substantially smaller than the viable cells. Therefore, at a given flow condition, viable cells can be focused at the channel centre whereas nonviable cells are focused along the sidewalls, enabling an efficient filtration of nonviable cells and debris as well as purification of viable cells.

To characterize the particle separation threshold in the developed sinusoidal channels, the commercially available polystyrene microbeads with average diameters of $3,5,8,10,13$, 15 and $20 \mu \mathrm{m}$ were tested. Figure 1(b) shows a colour-coded map for illustrating the particle focusing position and quality. The colours in the map indicate the occurrence frequency of particles at the specific lateral positions. Figure 1(b) indicates that the smaller particles have a wider flow range for the two-sided focusing pattern and a higher threshold of flow rate or Reynolds number for transforming the two-sided focusing pattern to single central focusing pattern. In contrast, larger particles possess a narrower flow range for the two-sided focusing pattern but a wider range for the single central focusing pattern. Besides, the threshold of flow rate of the transformation of the two-sided focusing pattern to the single central focusing 
pattern is much lower. This unique differential focusing phenomenon offers a promising overlapping flow region, where large particles and small particles focus at the channel centre and along two sidewalls, respectively. Subsequently, an efficient separation could be achieved by splitting the streamline at the end of the channel. The theory of particle differential inertial focusing and separation in sinusoidal channels is detailed in the supplementary information.
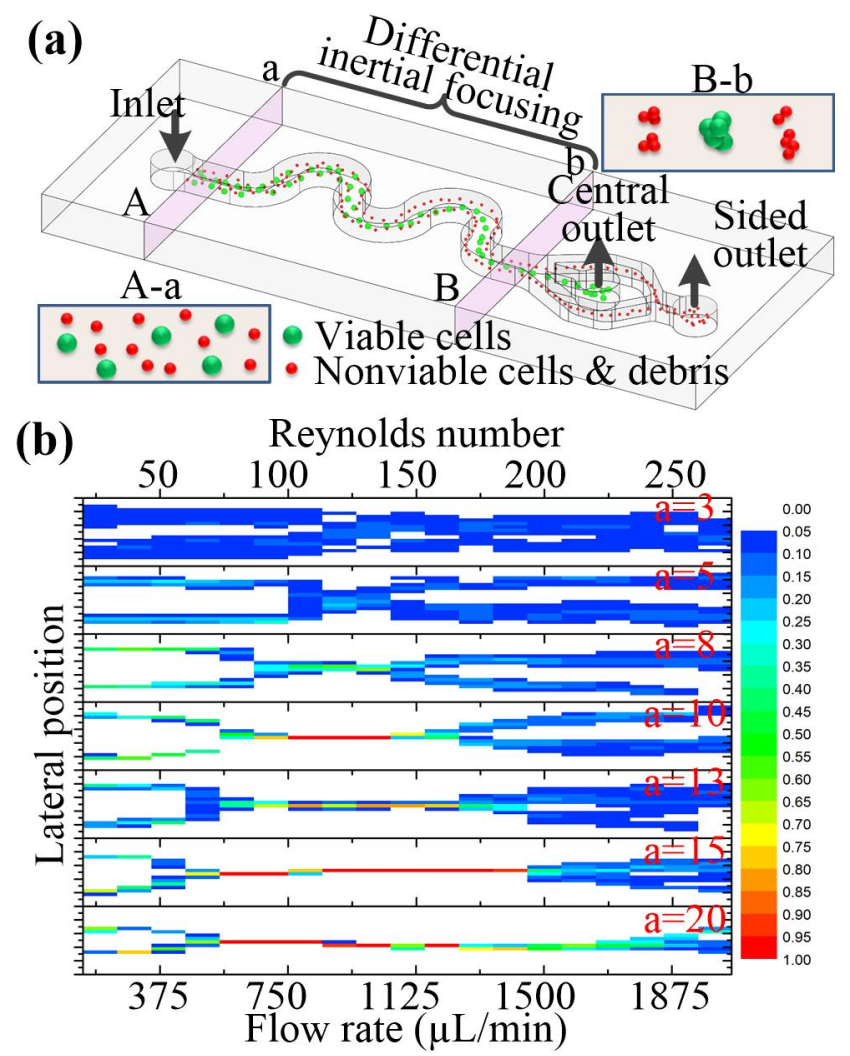

Figure 1 (a) Schematic illustration of separation of viable and nonviable cells by differential inertial focusing in the sinusoidal channel. Brightfield images of cells at the inlet (i) and outlet (ii) of the sinusoidal microchannel. (b) Colour-coded map illustrating inertial focusing of differently-sized particles (diameter $=3-20 \mu \mathrm{m}$ ) under various flow rates (or Dean number). The colours in the map indicate the normalized frequencies of particles at the specific lateral positions.

Microfluidic Purification of Floating Cancer Cells. Before processing the floating cancer cells, we measured the size distribution of viable cells, nonviable cells and debris in the supernatant medium, Figure 2(a). The acridine orange/ propidium iodide (AO/PI) viability assay was used to distinguish these cells, Figure 2(b). AO can stain all nucleated cells to show green fluorescence, whereas PI can only penetrate the dead cells to generate red fluorescence. Viable cells are therefore green in colour and dead cells are orange in colour 
because that the fluorescence of both AO and PI are merged. Most debris show no or weak fluorescence signal. The measured diameters of viable cells, nonviable cells and debris are 12.2 $\pm 3.2 \mu \mathrm{m}, 6.6 \pm 1.6 \mu \mathrm{m}$, and 2.6 $\pm 0.6 \mu \mathrm{m}$ (average diameter \pm standard deviation), respectively. This is because nonviable cells undergoing apoptosis, a programmed cell death, will shrink, lose cell volume or break into fragments, a ubiquitous characteristic of programmed cell death ${ }^{47}$. Thus, we can see an obvious size difference between viable cells, nonviable cells and debris. Eventually, this discrimination facilitates the size-dependent separation of viable cells and nonviable cells.

We tested the inertial focusing properties of floating cancer cells in the supernatant using the sinusoidal channels, supplementary information and Figure S2. We processed the floating cancer cells at an optimal flow rate of $600 \mu \mathrm{L} / \mathrm{min}(\mathrm{Re}=80)$ in the sinusoidal microchannel. Floating cancer cells before separation and cells collected by the two outlets after separation were respectively seeded in a 96-well-plate with fresh culture medium and incubated for 24 hours. Figure 2(c) shows the brightfield images of floating cancer cells at the inlet and at the two outlets just after separation ( 0 hours) and after 24 hours. Based on the red fluorescence spots, which indicate the nonviable cells stained by the PI, we can observe that there were much fewer dead cells in the central outlet than that of the inlet and sided outlet. Additionally, small non-fluorescent debris was concentrated at the sided outlet. This suggested that the nonviable cells and debris were both removed from the floating cancer cells, purifying the viable cells at the central outlet. After two circulatory processes, the purity of viable cells was increased from $19.9 \%$ to $76.6 \%$, with a recovery of $69.7 \%$.

Interestingly, after the incubation of 24 hours, floating cancer cells could adhere to the bottom surface of the well-plate and resumed proliferation, Figure 2(c). A colourimetric assay MTS was used to evaluate the cell viability by assessing the cellular metabolic activity before separation at the inlet and after separation at the two outlets. An ELISA reader was used to quantify the colourimetric optical density which is proportional to the viability of cells. The optical density at the central outlet was much higher than the inlet and the sided outlet, indicating that the viable cells were purified and concentrated at the central outlet, Figure 2(d). Moreover, a low optical density at the sided outlet demonstrated that nonviable cells and debris were concentrated and very few viable cells were collected. The results agree well with the brightfield images of cell culture, where dense adherent cells were retained at the central outlet after an incubation of 24 hours, Figure 2(c). 
It should be noted that the size of cells in the culture medium is not uniform but varies widely due to different stages of the cell cycle and apoptosis. Moreover, the size of viable and nonviable cells is partially overlapped. That may explain why a small portion of nonviable cells remain at the central outlet after processing, although their number is significantly reduced compared to the inlet, Figure 2(c). Around 30\% of viable cells are lost during the process, and most of them have a relatively small size, which is comparable to the dead cells. This is the intrinsic limitation of size-based purification approach. To achieve a better quality of viable cells, other separation approaches such as that based on the cell stiffness, electric property, metabolic activity or density may be combined with the current size-based approach.
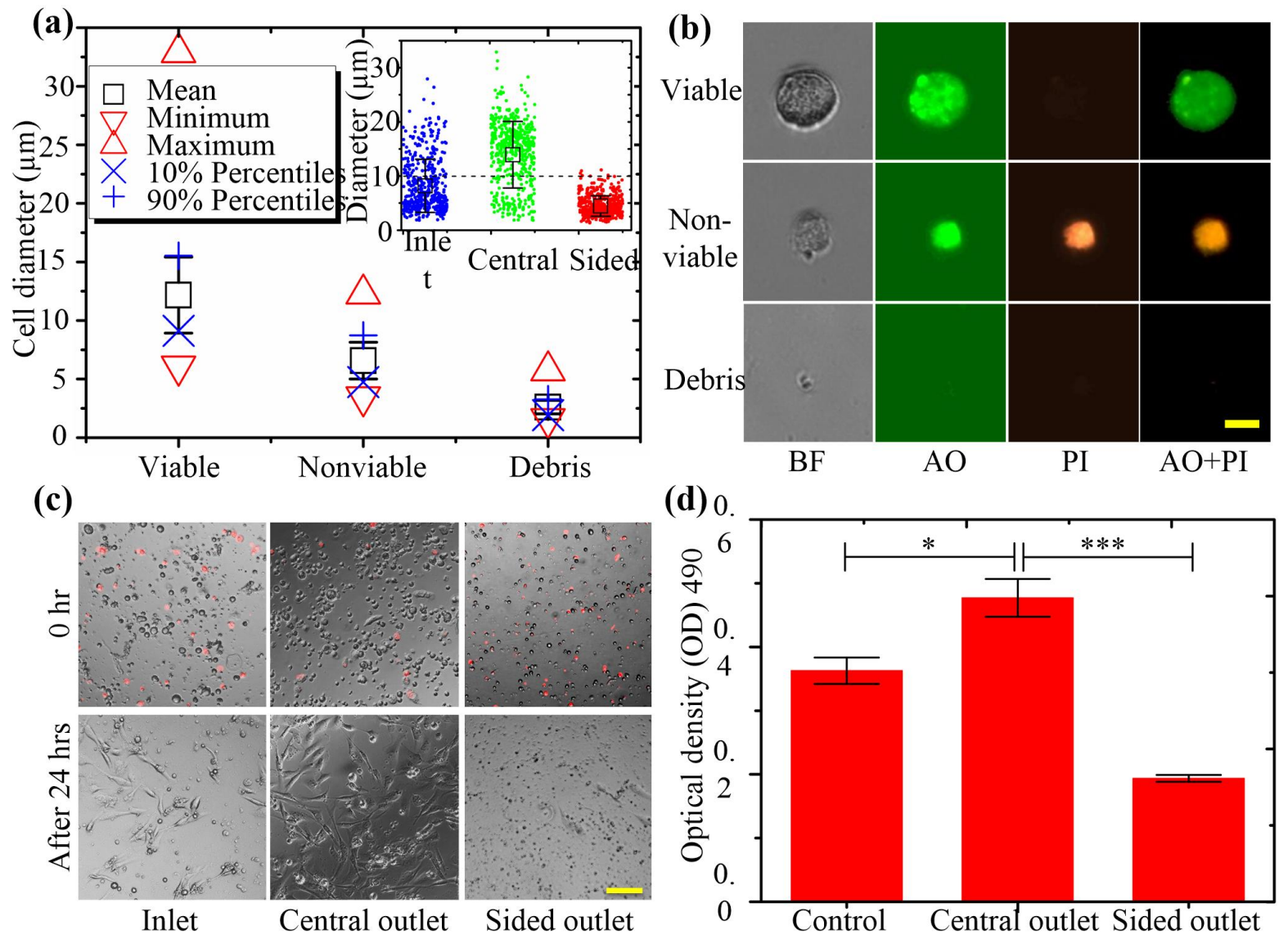

Figure 2 (a) Size distribution of viable cells, nonviable cells and debris. The insert shows the size distribution of the whole floating cells at the inlet (before the separation) and two outlets of central and sided branches (after separation). (b) The brightfield (BF) and fluorescent images (AO \& PI) of viable cells, nonviable cells and debris. Viable cells are in green colour and nonviable cells are in orange colour when merging both the fluorescence of AO and PI. The scale bar is $10 \mu \mathrm{m}$. (c) The brightfield images of floating cancer cells at the inlet and at the two outlets just after separation ( 0 hours) and after 24 hours incubation. The red dots indicate the nonviable cells which are stained by the PI. The scale bar is $100 \mu \mathrm{m}$. (d) In-vitro cell viability assay was performed using MTS to assess the cell metabolic activity at the inlet and the two outlets after 24 hours. A significant increment of 
optical density from the inlet to the central outlet indicates that the viable cells are purified and concentrated. * represents significance at probability $0.05 ; * * *$ represents significance at probability 0.001 .

After separation, we studied the cell proliferation of MDA-MB-231 cells, and the data revealed no significant difference between the adherent and floating MDA-MB-231 cells, supplementary information and Figure S3. Besides, we investigated the protrusive cellular structures such as the formation of filopodia and lamellipodia in both the floating and adherent cells by staining actin. Actin is regarded as the building block of cytoskeleton. During metastasis, actin filaments organize into elongated bundles and generate protrusive force to drive cell migration and invasion. ${ }^{48}$ The induction of filopodia and lamellipodia is crucial for facilitating the invasion and migration of cancer cells to the surrounding tissue. ${ }^{49,50}$ We found that the number of cells with the presence of filopodia and extensions of lamellipodia were both significantly higher in floating cells than the corresponding adherent cells, Figures S4. Furthermore, the relative expression of cyclooxygenase-2 (COX-2) in the floating and adherent MDA-MB-231 cells was measured. We found that the expression of COX-2 in floating MDA-MB-231 cells was significantly higher when compared to the adherent cells, Figure S5. COX-2 is related to the existence of epithelial-mesenchymal transition (EMT) changes in breast cancers. ${ }^{51}$ It is a crucial inflammatory enzyme that regulates prostaglandin synthesis $\left(\mathrm{PGE}_{2}\right)$ and also plays a role in tumour aggressiveness and invasiveness. ${ }^{52}$ Intriguingly, this new evidence of COX-2 regulation in floating MDA-MB231 cells may confer further understanding on the role of anoikis resistance and enhanced invasiveness.

Drug Screening of Adherent and Floating Cancer Cells. After purifying the floating cancer cells, we conducted drug screening with both the floating and adherent MDA-MB-231 cells respectively. In this work, Dox, a front-line chemotherapeutic agent for the treatment of a wide range of malignancies, was used to treat both cells. Figure 3(a) shows the overall procedure for drug screening with both cell types. Floating cancer cells from the culture supernatant were processed and purified by the proposed microfluidic device. The adherent 
cells were detached from the culture flask through trypsinization. Subsequently, both the floating and adherent cells were re-seeded in a 96-well-plate. The effects of Dox on floating and adherent MDA-MB-231 cells exposed to a serial dilution of Dox ranging from $0.01 \mu \mathrm{M}$ to $100 \mu \mathrm{M}$ for 24 hours were studied.

Interestingly, we found that floating cells showed a 30-fold acquired resistance as compared to adherent cells $\left(\mathrm{IC}_{50}=1.2 \mu \mathrm{M}\right.$ vs $\mathrm{IC}_{50}=31.45 \mu \mathrm{M}$ ), Figure 3(b). Furthermore, Figure 3(c) shows the brightfield images of floating and adherent cells after being treated by the same dose of $1 \mu \mathrm{M}$ Dox for 24 hours as well as without drug treatment. Here, the halfmaximal inhibitory concentration $\mathrm{IC}_{50}$ value, which is defined as the dose of the drug required to kill $50 \%$ of cancer cells, was determined using MTS assay. Drug screening results showed that floating MDA-MB-231 cells were inherently more chemoresistant than the adherent cells, Figure 3(b, c), which is consistent with the findings reported by Bizjak et al. ${ }^{53}$. A prerequisite for metastasis is the detachment of cancer cell from the extracellular matrix. The observed chemoresistance may be due to the presence of a small proportion of putative stem-like breast cancer cells. Such stemness was earlier reported in MDA-MB-231 cells upon Dox treatment ${ }^{54}$. In contrast, the inability of chemoresistant floating prostate LnCAP cells to form 3D spheroids excludes the presumption of breast cancer stem cell-mediated chemoresistance ${ }^{55}$. Nevertheless, EMT is a type of phenotypic transition that enhances the migratory potential of cancer cells ${ }^{55}$. Hence, it may be speculated that detachment of cells from the ECM by itself confers chemoresistance as EMT drives the metastatic cascade. However, the chemoresistance of floating MDA-MB-231 cells observed in this study may not be generalized as a driving force for metastasis, as adherent cells cultured under hypoxic conditions also display chemoresistance to anticancer drugs ${ }^{56}$. 
(a) Supernatant Microfluidic device Viable floating cells 96-well-plate
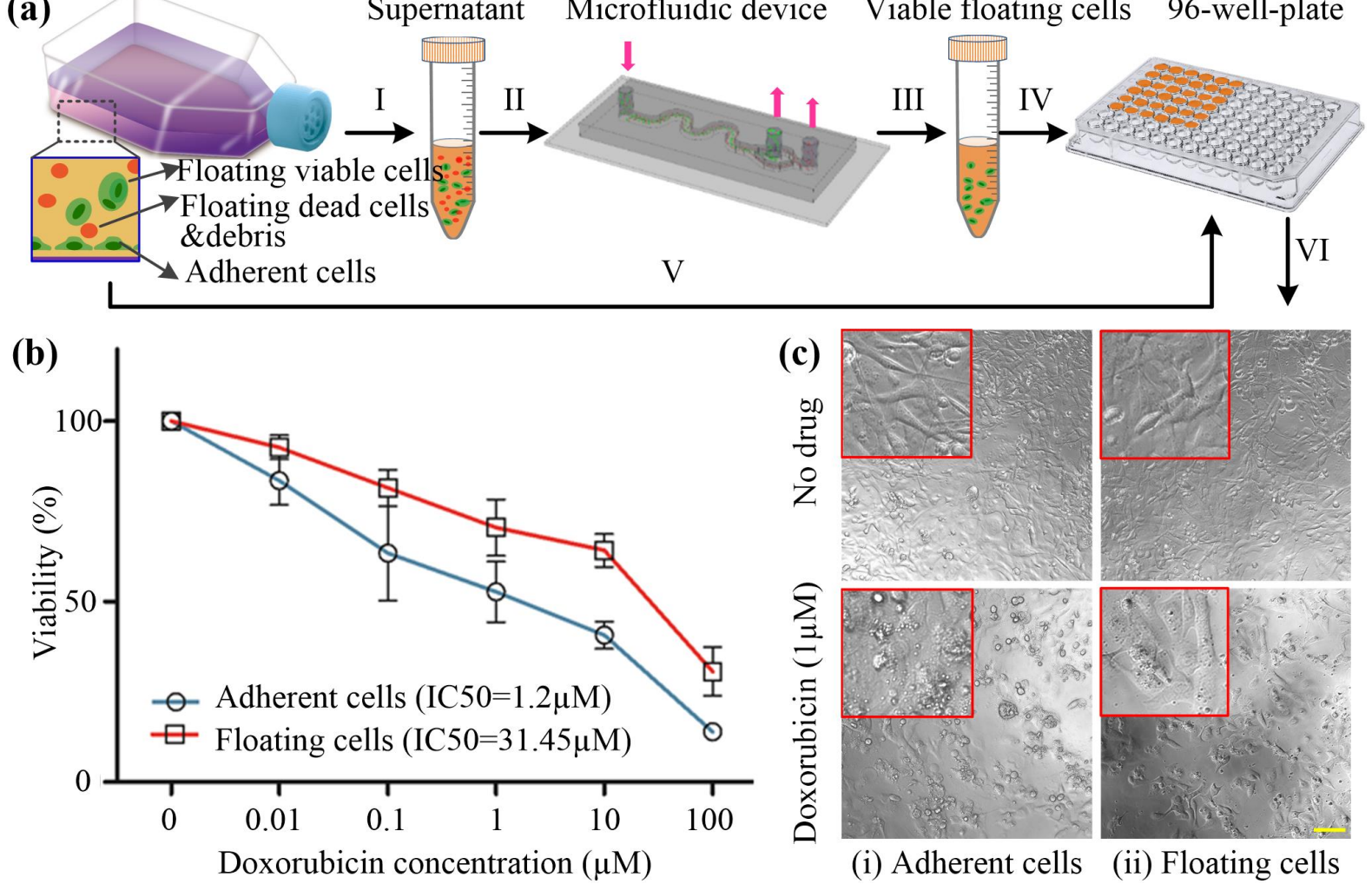

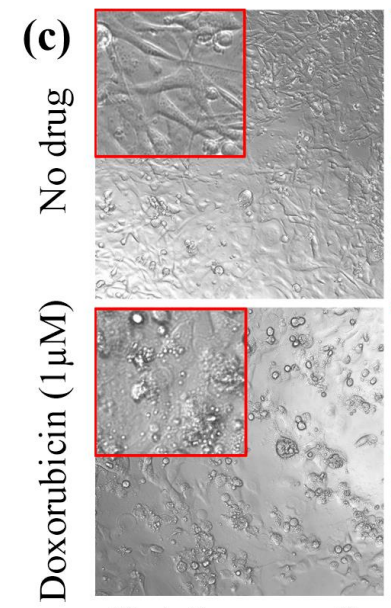

(i) Adherent cells

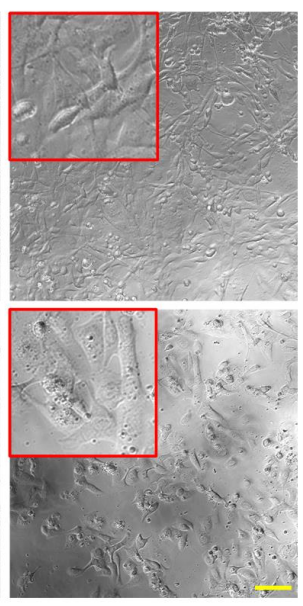

(ii) Floating cells

Figure 3. Effect of Dox on the viability of floating and adherent MDA-MB-231 cells. (a) The procedure for purification of floating cancer cells and drug testing of floating and adherent cancer cells, including (I) collection of supernatant, (II) infusion of cell suspension into the microfluidic device, (III) inertial separation of floating cells, (IV) seeding of floating cells, (V) trypsinization and reseeding of adherent cells, and (VI) drug screening. (b) The viability of floating and adherent cancer cells MDA-MB-231 after treatment with Dox concentrations of $0 \mu \mathrm{M}$ to $100 \mu \mathrm{M}$ for 24 hours. The half-maximal inhibitory concentration $\mathrm{IC}_{50}$ of adherent and floating cells is $1.2 \mu \mathrm{M}$ and $31.45 \mu \mathrm{M}$, respectively. (c) Brightfield microscopic images $(10 \times)$ of floating and adherent cancer cells MDA-MB-231 culture without and with $1 \mu \mathrm{M}$ Dox treatment for 24 hours. Inserts are the magnified images of cell culture. The scale bar is $100 \mu \mathrm{m}$.

\section{Three-dimensional Co-culture Model of Tumour/ and Stroma Spheroids Using Liquid}

Marbles. Multicellular three dimensional (3D) culture and interaction with stromal components, as a "more clinically relevant" tumour model, are of great importance for the study of tumour metastases, efficient drug screening during early drug development as well as the studies of pharmacological mechanisms of drugs or drug targets under the clinical development ${ }^{57}$. The standard monolayer two-dimensional (2D) cell cultures and xenograft 
models are believed to lack tumour complexity and are not physiologically relevant, and translation into in vivo may not be successful ${ }^{58,59}$. In epithelial tumours, fibroblasts are the most abundant stromal cell type ${ }^{60}$. Therefore, we introduced a 3D co-culture model of cancer cells and human mammary fibroblasts (HMF) using the liquid marble microbioreactor technique ${ }^{61}$ which can be used for studying cell metastases within 3D microenvironment.

Figure 4(a) illustrates the experimental procedure and Figure 4(b) shows the production, manipulation and culture of liquid marbles, and the results of co-culture of HMF spheroids and cancer cells. Both floating cancer cells and adherent cancer cells were tested and cocultured with the HMF spheroids using the liquid marbles, respectively. Over $24 \mathrm{~h}$ of incubation, both the floating and adherent cancer cells eventually adhered and dispersed into the fibroblast spheroid. It should be noted that the current work only introduced a conceptual framework for studying cancer cell metastases within 3D environment by using a novel bioreactor liquid marble technique, further studies are needed to quantify the metastatic characteristics of floating and adherent cancer cells. Besides, drug testing on 3D culture model may provide more accurate information than the $2 \mathrm{D}$ monolayer culture, since the tumour microenvironment strongly affects the drug sensitivity of cancer cells and consequently the efficacy of drug treatments, and 3D co-culture model could replicate the chemoresistance to treatment often observed in vivo ${ }^{62}$.

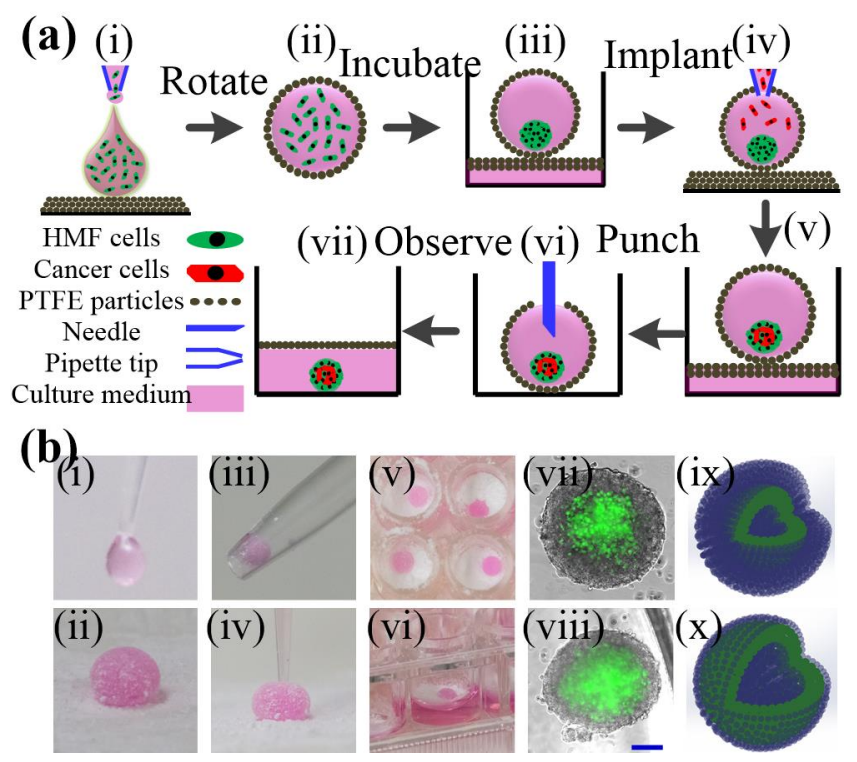

Figure 4. (a) Schematics of production of mammary fibroblasts (HMF) spheroids and coculture of HMF spheroids and cancer cells using the floating liquid marbles. (i) Dropping a $10-\mu \mathrm{L}$ culture medium with defined cell number onto the polytetrafluoroethylene (PTFE) 
powder bed; (ii) Coating the surface of liquid droplets with PTFE particles to form liquid marbles; (iii) Transferring the liquid marbles onto a double-layer configuration to float the liquid marbles for incubation of 24 hours, and the HMF spheroids can be formed; (iv) Implanting the MDA-MB-231 cancer cells with defined cell numbers into the liquid marbles containing the HMF spheroids; (v) Incubating the liquid marbles to co-culture cancer cells and HMF spheroids for 24 hours; (vi) Punching and breaking the liquid marbles; and (vii) The spheroids are released and sink into the bottom for observation. (b) Photos of production, manipulation and culture of liquid marbles. (i) Production of a droplet by a pipette; (ii) A liquid marble on the PTFE powder bed; (iii) Pick up and transfer the liquid marble by a flattened pipette tip; (iv) Penetrate the liquid marble to implant cancer cells; (v-vi) Top and side views of liquid marbles floating on a double-layer configuration; (vii-viii) The floating and adherent MDA-MB-231 cells adhere and disperse into the HMF spheroids; (ix-x) 3D schematic diagrams of co-culture of floating and adherent cancer cells with HMF spheroids. The scale bar is $100 \mu \mathrm{m}$. The cancer cells are stained by MitoTracker green dye to track their positions in the spheroids.

\section{CONCLUSIONS}

This paper reports an efficient microfluidic method for purifying viable floating cancer cells. The purified floating cells were further characterized by drug screening and 3D cell coculture model. The linear sinusoidal channels focus floating viable and dead cells/debris differentially along the lateral direction. The purity of viable cells was increased significantly from $19.9 \%$ to $76.6 \%$, with a recovery of $\sim 70 \%$ after two circulatory processes. Both the floating and adherent MDA-MB-231 cells were tested using a front-line chemotherapeutic drug Dox. Results showed that floating MDA-MB-231 cells are more drug-resistant than the corresponding adherent cells, possessing 30-fold acquired chemoresistance (IC 50 -floating $=31.45$ $\mu \mathrm{M}$; IC50-adherent $=1.2 \mu \mathrm{M}$ ). In addition, we studied the cell proliferation, protrusive cellular structures and the expression of COX-2 in both the floating and adherent MDA-MB-231 cells. Furthermore, we developed a conceptual framework for studying cell metastatic characteristics within 3D microenvironment by using the floating liquid marble-based spheroid model.

\section{CONFLICT OF INTEREST}

The authors declare that they have no conflict of interest. 


\section{SUPPORTING INFORMATION}

S-1 Theory of particle differential inertial focusing and separation in sinusoidal channels.

S-2 Calculation of purity and recovery.

S-3. Inertial focusing of floating cancer cells in the sinusoidal channels at different flow rates.

S-4. Cell proliferation of adherent and floating MDA-MB-231 cells.

S-5. Morphological quantification of cytoskeletal changes in floating and adherent MDAMB-231 breast cancer cells.

S-6. Quantification of COX-2 in floating and adherent MDA-MB-231 breast cancer cells.

Figure S1. The Operational map of the $\mathrm{F}_{\mathrm{L}} / \mathrm{F}_{\mathrm{D}}$ scaling factor $\omega=\left(\mathrm{a} / \mathrm{D}_{\mathrm{h}}\right) / \operatorname{Re}^{1 / 2} \sim$ particle blockage ratio $\left(\mathrm{a} / \mathrm{D}_{\mathrm{h}}\right)$ for the sinusoidal microchannel ( $\left.\mathrm{W}=200 \mu \mathrm{m}, \mathrm{H}=50 \mu \mathrm{m}, \mathrm{R}=250 \mu \mathrm{m}\right)$.

Figure S2. Brightfield images of floating cancer cell inertial focusing in the serpentine channels under different flow conditions.

Figure S3. (a) Profile of DAPI-fluorescent measurement. Student's t test was performed, $n=6$

(b) Western blot analysis of the expression of PCNA in adherent and floating MDA-MB-231 breast cancer cells.

Figure S4. Quantification of the number of filopodia and lamellipodia by actin cytoskeleton staining.

Figure S5. Western blot analysis of the expression of COX-2 in adherent and floating MDAMB-2312 breast cancer cells.

\section{ACKNOWLEDGEMENTS}

J. Zhang greatly acknowledges the support from the National Natural Science Foundation of China (Grant No.51705257), the Natural Science Foundation of Jiangsu Province (Grant No. BK20170839), and the Griffith University New Research Grant 2020. N.T. Nguyen and W.H. Li acknowledge the support from the Australian Research Council (ARC) Discovery Project (Grant No. DP180100055). R. Vadivelu and D. Yuan are recipients of the International Postdoctoral Fellowship of Japan Society for the Promotion of Science.

\section{REFERENCES}

(1) Hanahan, D.; Weinberg, R. A. Hallmarks of cancer: the next generation. Cell 2011, 144, 646-674.

(2) Smid, M.; Wang, Y.; Zhang, Y.; Sieuwerts, A. M.; Yu, J.; Klijn, J. G.; Foekens, J. A.; Martens, J. W. Subtypes of breast cancer show preferential site of relapse. Cancer Res. 2008, $68,3108-3114$. 
(3) Pani, G.; Galeotti, T.; Chiarugi, P. Metastasis: cancer cell's escape from oxidative stress. Cancer Metastasis Rev. 2010, 29, 351-378.

(4) Jones, S. E. Metastatic breast cancer: the treatment challenge. Clin. Breast Cancer 2008, 8 , 224-233.

(5) Esparza-López, J.; Alvarado-Muñoz, J. F.; Escobar-Arriaga, E.; Ulloa-Aguirre, A.; de Jesús Ibarra-Sánchez, M. Metformin reverses mesenchymal phenotype of primary breast cancer cells through STAT3/NF-kB pathways. BMC Cancer 2019, 19, 728.

(6) Frisch, S. M.; Ruoslahti, E. Integrins and anoikis. Curr. Opin. Cell Biol. 1997, 9, 701-706.

(7) Boudreau, N. J.; Jones, P. L. Extracellular matrix and integrin signalling: the shape of things to come. Biochem. J. 1999, 339, 481-488.

(8) Reddig, P. J.; Juliano, R. L. Clinging to life: cell to matrix adhesion and cell survival. Cancer Metastasis Rev. 2005, 24, 425-439.

(9) Giancotti, F. G.; Ruoslahti, E. Integrin signaling. Science 1999, 285, 1028-1033.

(10) Jenning, S.; Pham, T.; Ireland, S. K.; Ruoslahti, E.; Biliran, H. Bit1 in anoikis resistance and tumor metastasis. Cancer Lett. 2013, 333, 147-151.

(11) Akekawatchai, C.; Roytrakul, S.; Kittisenachai, S.; Isarankura-Na-Ayudhya, P.; Jitrapakdee, S. Protein profiles associated with Anoikis resistance of metastatic MDA-MB231 breast cancer cells. Asian Pac. J. Cancer Prev. 2016, 17, 581-590.

(12) Paoli, P.; Giannoni, E.; Chiarugi, P. Anoikis molecular pathways and its role in cancer progression. BBA-Mol Cell Res. 2013, 1833, 3481-3498.

(13) Glinskii, A. B.; Smith, B. A.; Jiang, P.; Li, X.-M.; Yang, M.; Hoffman, R. M.; Glinsky, G. V. Viable circulating metastatic cells produced in orthotopic but not ectopic prostate cancer models. Cancer Res. 2003, 63, 4239-4243.

(14) Kim, Y.-N.; Koo, K. H.; Sung, J. Y.; Yun, U.-J.; Kim, H. Anoikis resistance: an essential prerequisite for tumor metastasis. Int. J. Cell Biol. 2012, $2012,306879$.

(15) Malin, D.; Strekalova, E.; Petrovic, V.; Rajanala, H.; Sharma, B.; Ugolkov, A.; Gradishar, W. J.; Cryns, V. L. ERK-regulated $\alpha \mathrm{B}$-crystallin induction by matrix detachment inhibits anoikis and promotes lung metastasis in vivo. Oncogene 2015, 34, 5626-5634.

(16) Thompson, E. W.; Paik, S.; Brünner, N.; Sommers, C. L.; Zugmaier, G.; Clarke, R.; Shima, T. B.; Torri, J.; Donahue, S.; Lippman, M. E. Association of increased basement membrane invasiveness with absence of estrogen receptor and expression of vimentin in human breast cancer cell lines. J. Cell. Physiol. 1992, 150, 534-544.

(17) Culty, M.; Shizari, M.; Thompson, E. W.; Underhill, C. B. Binding and degradation of hyaluronan by human breast cancer cell lines expressing different forms of CD44: correlation with invasive potential. J. Cell. Physiol. 1994, 160, 275-286.

(18) Holliday, D. L.; Speirs, V. Choosing the right cell line for breast cancer research. Breast Cancer Res. 2011, 13, 215.

(19) Jin, X.; Mu, P. Targeting breast cancer metastasis. Breast Cancer (Auckl) 2015, 9, BCBCR. S25460.

(20) Mori, S.; Chang, J. T.; Andrechek, E. R.; Matsumura, N.; Baba, T.; Yao, G.; Kim, J. W.; Gatza, M.; Murphy, S.; Nevins, J. R. Anchorage-independent cell growth signature identifies tumors with metastatic potential. Oncogene 2009, 28, 2796-2805.

(21) Mansilla, S.; Priebe, W.; Portugal, J. Mitotic catastrophe results in cell death by caspasedependentand caspase-independent mechanisms. Cell Cycle 2006, 5, 53-60.

(22) Palorini, R.; Votta, G.; Pirola, Y.; De Vitto, H.; De Palma, S.; Airoldi, C.; Vasso, M.; Ricciardiello, F.; Lombardi, P. P.; Cirulli, C. Protein kinase A activation promotes cancer cell resistance to glucose starvation and anoikis. PLoS Genet. 2016, 12, e1005931.

(23) Sánchez-Alcázar, J. A.; Khodjakov, A.; Schneider, E. Anticancer drugs induce increased mitochondrial cytochrome c expression that precedes cell death. Cancer Res. 2001, 61, 10381044. 
(24) Islam, M.; Brink, H.; Blanche, S.; DiPrete, C.; Bongiorno, T.; Stone, N.; Liu, A.; Philip, A.; Wang, G.; Lam, W. Microfluidic sorting of cells by viability based on differences in cell stiffness. Sci. Rep. 2017, 7, 1997.

(25) Gossett, D. R.; Weaver, W. M.; Mach, A. J.; Hur, S. C.; Tse, H. T. K.; Lee, W.; Amini, H.; Di Carlo, D. Label-free cell separation and sorting in microfluidic systems. Anal. Bioanal. Chem. 2010, 397, 3249-3267.

(26) Kumar, A.; Bhardwaj, A. Methods in cell separation for biomedical application: cryogels as a new tool. Biomed Mater. 2008, 3, 034008.

(27) Shafiee, H.; Sano, M. B.; Henslee, E. A.; Caldwell, J. L.; Davalos, R. V. Selective isolation of live/dead cells using contactless dielectrophoresis (cDEP). Lab Chip 2010, 10, 438-445.

(28) Yildizhan, Y.; Erdem, N.; Islam, M.; Martinez-Duarte, R.; Elitas, M. Dielectrophoretic separation of live and dead monocytes using 3D carbon-electrodes. Sensors 2017, 17, 2691.

(29) Patel, S.; Showers, D.; Vedantam, P.; Tzeng, T.-R.; Qian, S.; Xuan, X. Microfluidic separation of live and dead yeast cells using reservoir-based dielectrophoresis. Biomicrofluid 2012, 6, 034102.

(30) Zalis, M. C.; Reyes, J. F.; Augustsson, P.; Holmqvist, S.; Roybon, L.; Laurell, T.; Deierborg, T. Label-free concentration of viable neurons, hESCs and cancer cells by means of acoustophoresis. Integr. Biol. 2016, 8, 332-340.

(31) Yang, A. H.; Soh, H. T. Acoustophoretic sorting of viable mammalian cells in a microfluidic device. Anal. Chem. 2012, 84, 10756-10762.

(32) Tottori, N.; Nisisako, T.; Park, J.; Yanagida, Y.; Hatsuzawa, T. Separation of viable and nonviable mammalian cells using a deterministic lateral displacement microfluidic device. Biomicrofluid 2016, 10, 014125.

(33) Pan, C. W.; Horvath, D. G.; Braza, S.; Moore, T.; Lynch, A.; Feit, C.; Abbyad, P. Sorting by interfacial tension (SIFT): label-free selection of live cells based on single-cell metabolism. Lab Chip 2019, 19, 1344-1351.

(34) Kwon, T.; Yao, R.; Hamel, J.-F. P.; Han, J. Continuous removal of small nonviable suspended mammalian cells and debris from bioreactors using inertial microfluidics. Lab Chip 2018, 18, 2826-2837.

(35) Whitesides, G. M.; Ostuni, E.; Takayama, S.; Jiang, X.; Ingber, D. E. Soft lithography in biology and biochemistry. Annu. Rev. Biomed. Eng. 2001, 3, 335-373.

(36) Nguyen, N.-T.; Wereley, S. T.; Shaegh, S. A. M. Fundamentals and applications of microfluidics; Artech house, 2019.

(37) Zhang, J.; Yuan, D.; Zhao, Q.; Teo, A. J.; Yan, S.; Ooi, C. H.; Li, W.; Nguyen, N.-T. Fundamentals of differential particle inertial focusing in symmetric sinusoidal microchannels. Anal. Chem. 2019, 91, 4077-4084.

(38) Carvalho, C.; Santos, R. X.; Cardoso, S.; Correia, S.; Oliveira, P. J.; Santos, M. S.; Moreira, P. I. Doxorubicin: the good, the bad and the ugly effect. Curr. Med. Chem. 2009, 16, 3267-3285.

(39) Gordon, A. N.; Fleagle, J. T.; Guthrie, D.; Parkin, D. E.; Gore, M. E.; Lacave, A. J. Recurrent epithelial ovarian carcinoma: a randomized phase III study of pegylated liposomal doxorubicin versus topotecan. J. Clin. Oncol. 2001, 19, 3312-3322.

(40) Lovitt, C. J.; Shelper, T. B.; Avery, V. M. Doxorubicin resistance in breast cancer cells is mediated by extracellular matrix proteins. BMC Cancer 2018, 18, 41.

(41) Tacar, O.; Sriamornsak, P.; Dass, C. R. Doxorubicin: an update on anticancer molecular action, toxicity and novel drug delivery systems. J. Pharm. Pharmacol. 2013, 65, 157-170.

(42) Hilmer, S. N.; Cogger, V. C.; Muller, M.; Le Couteur, D. G. The hepatic pharmacokinetics of doxorubicin and liposomal doxorubicin. Drug Metab. Dispos. 2004, 32, 794-799. 
(43) Tewey, K.; Rowe, T.; Yang, L.; Halligan, B.; Liu, L.-F. Adriamycin-induced DNA damage mediated by mammalian DNA topoisomerase II. Science 1984, 226, 466-468.

(44) Campiglio, M.; Somenzi, G.; Olgiati, C.; Beretta, G.; Balsari, A.; Zaffaroni, N.; Valagussa, P.; Ménard, S. Role of proliferation in HER2 status predicted response to doxorubicin. Int. J. Cancer 2003, 105, 568-573.

(45) Gewirtz, D. A critical evaluation of the mechanisms of action proposed for the antitumor effects of the anthracycline antibiotics adriamycin and daunorubicin. Biochem. Pharmacol. 1999, 57, 727-741.

(46) Minotti, G.; Menna, P.; Salvatorelli, E.; Cairo, G.; Gianni, L. Anthracyclines: molecular advances and pharmacologic developments in antitumor activity and cardiotoxicity. Pharmacol. Rev. 2004, 56, 185-229.

(47) Kerr, J. F. R.; Wyllie, A. H.; Currie, A. R. Apoptosis: A Basic Biological Phenomenon with Wideranging Implications in Tissue Kinetics. Br. J. Cancer 1972, 26, 239-257.

(48) Machesky, L. M. Lamellipodia and filopodia in metastasis and invasion. FEBS Lett. 2008, 582, 2102-2111.

(49) Svitkina, T. The actin cytoskeleton and actin-based motility. Cold Spring Harb. Perspect. Biol. 2018, 10, a018267.

(50) Stevenson, R. P.; Veltman, D.; Machesky, L. M. Actin-bundling proteins in cancer progression at a glance. J. Cell Sci. 2012, 125, 1073-1079.

(51) Jang, T. J.; Jeon, K. H.; Jung, K. H. Cyclooxygenase-2 expression is related to the epithelial-to-mesenchymal transition in human colon cancers. Yonsei Med. J. 2009, 50, 818824.

(52) Howe, L.; Subbaramaiah, K.; Brown, A.; Dannenberg, A. Cyclooxygenase-2: a target for the prevention and treatment of breast cancer. Endocr.-Relat. Cancer 2001, 8, 97-114.

(53) Bizjak, M.; Malavašič, P.; Dolinar, K.; Pohar, J.; Pirkmajer, S.; Pavlin, M. Combined treatment with Metformin and 2-deoxy glucose induces detachment of viable MDA-MB-231 breast cancer cells in vitro. Sci. Rep. 2017, 7, 1761.

(54) Bandyopadhyay, A.; Wang, L.; Agyin, J.; Tang, Y.; Lin, S.; Yeh, I.-T.; De, K.; Sun, L.Z. Doxorubicin in combination with a small TGF $\beta$ inhibitor: a potential novel therapy for metastatic breast cancer in mouse models. PLoS One 2010, 5, e10365.

(55) Kaushik, V.; Yakisich, J. S.; Way, L. F.; Azad, N.; Iyer, A. K. Chemoresistance of cancer floating cells is independent of their ability to form 3D structures: Implications for anticancer drug screening. J. Cell. Physiol. 2019, 234, 4445-4453.

(56) Karakashev, S. V.; Reginato, M. J. Progress toward overcoming hypoxia-induced resistance to solid tumor therapy. Cancer Manag. Res. 2015, 7, 253-264.

(57) Denayer, T.; Stöhr, T.; Van Roy, M. Animal models in translational medicine: Validation and prediction. New Horiz. Transl. Med. 2014, 2, 5-11.

(58) Unger, C.; Kramer, N.; Walzl, A.; Scherzer, M.; Hengstschlaeger, M.; Dolznig, H. Modeling human carcinomas: physiologically relevant 3D models to improve anti-cancer drug development. Adv. Drug Delivery. Rev. 2014, 79, 50-67.

(59) Jeong, S.-Y.; Lee, J.-H.; Shin, Y.; Chung, S.; Kuh, H.-J. Co-culture of tumor spheroids and fibroblasts in a collagen matrix-incorporated microfluidic chip mimics reciprocal activation in solid tumor microenvironment. PLoS One 2016, 11, e0159013.

(60) Kalluri, R. The biology and function of fibroblasts in cancer. Nat. Rev. Cancer 2016, 16, 582-598.

(61) Vadivelu, R. K.; Ooi, C. H.; Yao, R.-Q.; Velasquez, J. T.; Pastrana, E.; Diaz-Nido, J.; Lim, F.; Ekberg, J. A.; Nguyen, N.-T.; St John, J. A. Generation of three-dimensional multiple spheroid model of olfactory ensheathing cells using floating liquid marbles. Sci. Rep. 2015, 5, 15083. 
(62) Lazzari, G.; Nicolas, V.; Matsusaki, M.; Akashi, M.; Couvreur, P.; Mura, S. Multicellular spheroid based on a triple co-culture: A novel 3D model to mimic pancreatic tumor complexity. Acta Biomater. 2018, 78, 296-307. 


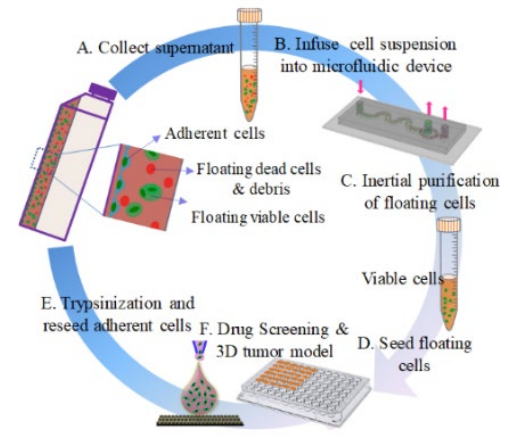

For TOC only 